\title{
A Universal Algorithm for Multivariate Integration
}

\author{
David Krieg \\ Mathematisches Institut, Universität Jena \\ Ernst-Abbe-Platz 2, 07743 Jena, Germany \\ email: david.krieg@uni-jena.de \\ and \\ Erich Novak \\ Mathematisches Institut, Universität Jena \\ Ernst-Abbe-Platz 2, 07743 Jena, Germany \\ email: erich.novak@uni-jena.de
}

January 30, 2016

\begin{abstract}
We present an algorithm for multivariate integration over cubes that is unbiased and has optimal order of convergence (in the randomized sense as well as in the worst case setting) for all Sobolev spaces $H^{r, \operatorname{mix}}\left([0,1]^{d}\right)$ and $H^{s}\left([0,1]^{d}\right)$ for $s>d / 2$.
\end{abstract}

AMS classification: 65D30, 65C05, 65Y20, 68Q25.

Short title: A Universal Algorithm for Integration

Key words: multivariate integration, randomized Frolov algorithm, universality, optimal order of convergence

Communicated by Andrew Stuart

Corresponding author: David Krieg 


\section{Introduction}

We present a new algorithm

$$
A_{n}(f)=\sum_{i=1}^{n} a_{i} f\left(x_{i}\right)
$$

for the approximation of integrals

$$
I_{d}(f)=\int_{[0,1]^{d}} f(x) \mathrm{d} x .
$$

Fred Hickernell wrote a paper "My dream quadrature rule" where he proposed five criteria that an ideal or "dream" quadrature formula should satisfy. We also present a list of five (similar, but different) properties of our "dream algorithm":

(P1) The algorithm $A_{n}$ should be an unbiased randomized algorithm, i.e.,

$$
\mathbb{E}\left(A_{n}(f)\right)=I_{d}(f)
$$

for all integrable functions. Of course this means that the weights $a_{i} \in \mathbb{R}$ and the points $x_{i} \in[0,1]^{d}$ are random variables. It is beneficial to have positive weights $a_{i} \geq 0$ for all $i$.

(P2) The randomized error

$$
\mathbb{E}\left(\left|A_{n}(f)-I_{d}(f)\right|\right)
$$

of $A_{n}$ should be small and/or optimal in the sense of order of convergence for "many" different classes of functions. In particular, we would like to have

$$
\mathbb{E}\left(\left|A_{n}(f)-I_{d}(f)\right|\right) \leq c_{r, d} n^{-r-1 / 2}(\log n)^{(d-1) / 2}\|f\|_{H^{r, \operatorname{mix}\left([0,1]^{d}\right)}}
$$

for all $r \in \mathbb{N}$, as well as for all $s \in \mathbb{N}$ with $s>d / 2$

$$
\mathbb{E}\left(\left|A_{n}(f)-I_{d}(f)\right|\right) \leq c_{s, d} n^{-s / d-1 / 2}\|f\|_{H^{s}\left([0,1]^{d}\right)} .
$$

(P3) The worst case error

$$
\sup _{\omega}\left|A_{n}^{\omega}(f)-I_{d}(f)\right|
$$

among the realizations $A_{n}^{\omega}$ of $A_{n}$ should be small and/or optimal in the sense 
of order of convergence for "many" different classes of functions, in particular

$$
\sup _{\omega}\left(\left|A_{n}^{\omega}(f)-I_{d}(f)\right|\right) \leq c_{r, d} n^{-r}(\log n)^{(d-1) / 2}\|f\|_{H^{r, \operatorname{mix}\left([0,1]^{d}\right)}}
$$

for all $r \in \mathbb{N}$, as well as for all $s \in \mathbb{N}$ with $s>d / 2$

$$
\sup _{\omega}\left(\left|A_{n}^{\omega}(f)-I_{d}(f)\right|\right) \leq c_{s, d} n^{-s / d}\|f\|_{H^{s}\left([0,1]^{d}\right)}
$$

(P4) The algorithm should have good tractability properties in the sense of the theory of "tractability of multivariate problems", see [5].

(P5) The algorithm should be easy to implement.

In this paper we concentrate on properties (P1), (P2) and (P3) and hence we are not specific on (P4) and (P5) and leave them for further research. In particular, we do not discuss tractability and all constants $c>0$ may depend on the dimension $d$ and the smoothness $r$ or $s$. A few remarks are in order.

1. The simplest Monte Carlo method certainly satisfies (P1). Therefore it is easy to run the algorithm a few times and to do an (a posteriori) error analysis. This is a great advantage of an unbiased algorithm. Of course the low rate $n^{-1 / 2}$ (even for very smooth integrands) is a big disadvantage of the simplest Monte Carlo method. Randomized algorithms with a higher rate of convergence are known and often they are unbiased; usually they are designed for a specific class of functions.

2. We do not know of any algorithm in the literature that satisfies (P2), even in the univariate case $d=1$. The upper bound (11) seems to be new. The main term $n^{-r-1 / 2}$ is of course optimal.

The bounds (2)-(4) are known and it is also known that they are optimal. The bound (3) is from Frolov, see [1, 8, 9]. The bounds (2) and (4) are from Bakhvalov and can be found in [4].

3. Many known algorithms (such as the Gaussian quadrature formulas) satisfy (P3) in the univariate case. It is also known that (modifications of) the Frolov algorithm satisfy (P3) for arbitrary $d$. Hence the Frolov algorithm (or some modifications of it) is "universal" in the worst case setting, see also the recent paper [10]. Since it is a deterministic algorithm it certainly cannot 
satisfy (P1) or (P2). The problem with any deterministic algorithm $A_{n}$ is that a computation of $A_{n}(f)$ does not come together with an error bound since usually the norm of $f$ is not known.

4. We did not discuss the property "extensible" in the list of Hickernell. We believe that this is another nice property but not as important as the other properties since it can decrease the total computing time only slightly.

In this paper we present an algorithm $\widetilde{M}_{a, B}$ with positive weights that satisfies (P1) and (P2) and (P3), see Section 5. In particular we prove the existence of $A_{n}$ such that (1) holds.

\section{Some Notation}

For $r, d \in \mathbb{N}$ the tensor product Sobolev space $H^{r, \operatorname{mix}}\left(\mathbb{R}^{d}\right)$ is defined as the space

$$
H^{r, \operatorname{mix}}\left(\mathbb{R}^{d}\right)=\left\{f \in L^{2}\left(\mathbb{R}^{d}\right) \mid D^{\alpha} f \in L^{2}\left(\mathbb{R}^{d}\right) \text { for every } \alpha \in\{0, \ldots, r\}^{d}\right\}
$$

of real valued functions, equipped with the scalar product

$$
\langle f, g\rangle_{H^{r, \operatorname{mix}\left(\mathbb{R}^{d}\right)}}=\sum_{\alpha \in\{0, \ldots, r\}^{d}}\left\langle D^{\alpha} f, D^{\alpha} g\right\rangle_{L^{2}\left(\mathbb{R}^{d}\right)}
$$

and hence with the norm

$$
\|f\|_{H^{r, \operatorname{mix}\left(\mathbb{R}^{d}\right)}}=\left(\sum_{\alpha \in\{0, \ldots, r\}^{d}}\left\|D^{\alpha} f\right\|_{L^{2}\left(\mathbb{R}^{d}\right)}^{2}\right)^{1 / 2} .
$$

It is known that $H^{r, m i x}\left(\mathbb{R}^{d}\right)$ is a Hilbert space and its elements can be taken to be continuous functions. In this paper, the Fourier transform is the unique continuous linear map $\hat{\imath}: L_{2}\left(\mathbb{R}^{d}\right) \rightarrow L_{2}\left(\mathbb{R}^{d}\right)$ with

$$
\hat{f}(y)=\int_{\mathbb{R}^{d}} f(x) e^{-2 \pi i\langle x, y\rangle} \mathrm{d} x
$$

for integrable $f$ and $y \in \mathbb{R}^{d}$. The space $H^{r, \operatorname{mix}}\left(\mathbb{R}^{d}\right)$ contains exactly those functions $f \in L^{2}\left(\mathbb{R}^{d}\right)$ with $\hat{f} \cdot h_{r}^{1 / 2} \in L^{2}\left(\mathbb{R}^{d}\right)$ for the Fourier transform $\hat{f}$ of $f$ and the weight 
function

$$
h_{r}: \mathbb{R}^{d} \rightarrow \mathbb{R}^{+}, \quad h_{r}(x)=\sum_{\alpha \in\{0, \ldots, r\}^{d}} \prod_{j=1}^{d}\left|2 \pi x_{j}\right|^{2 \alpha_{j}}=\prod_{j=1}^{d} \sum_{k=0}^{r}\left|2 \pi x_{j}\right|^{2 k}
$$

In terms of its Fourier transform, the norm of $f \in H^{r, \operatorname{mix}}\left(\mathbb{R}^{d}\right)$ is given by

$$
\|f\|_{H^{r, \text { mix }\left(\mathbb{R}^{d}\right)}}^{2}=\int_{\mathbb{R}^{d}}|\hat{f}(x)|^{2} \cdot h_{r}(x) \mathrm{d} x .
$$

Analogously, for $s, d \in \mathbb{N}$ the isotropic Sobolev space $H^{s}\left(\mathbb{R}^{d}\right)$ is the space

$$
H^{s}\left(\mathbb{R}^{d}\right)=\left\{f \in L^{2}\left(\mathbb{R}^{d}\right) \mid D^{\alpha} f \in L^{2}\left(\mathbb{R}^{d}\right) \text { for every } \alpha \in \mathbb{N}_{0}^{d} \text { with }\|\alpha\|_{1} \leq s\right\}
$$

of real valued functions, equipped with the scalar product

$$
\langle f, g\rangle_{H^{s}\left(\mathbb{R}^{d}\right)}=\sum_{\|\alpha\|_{1} \leq s}\left\langle D^{\alpha} f, D^{\alpha} g\right\rangle_{L^{2}\left(\mathbb{R}^{d}\right)}
$$

and hence with the norm

$$
\|f\|_{H^{s}\left(\mathbb{R}^{d}\right)}=\left(\sum_{\|\alpha\|_{1} \leq s}\left\|D^{\alpha} f\right\|_{L^{2}\left(\mathbb{R}^{d}\right)}^{2}\right)^{1 / 2} .
$$

This also defines a Hilbert space. In the following, let $s>d / 2$. Then $H^{s}\left(\mathbb{R}^{d}\right)$ also consists of continuous functions, exactly those functions $f \in L^{2}\left(\mathbb{R}^{d}\right)$ with $\hat{f} \cdot v_{s}^{1 / 2} \in L^{2}\left(\mathbb{R}^{d}\right)$ for the Fourier transform $\hat{f}$ of $f$ and the weight function

$$
v_{s}: \mathbb{R}^{d} \rightarrow \mathbb{R}^{+}, \quad v_{s}(x)=\sum_{\|\alpha\|_{1} \leq s} \prod_{j=1}^{d}\left|2 \pi x_{j}\right|^{2 \alpha_{j}} \asymp\left(1+\|x\|_{2}^{2}\right)^{s} .
$$

In terms of its Fourier transform, the norm of $f \in H^{s}\left(\mathbb{R}^{d}\right)$ is given by

$$
\|f\|_{H^{s}\left(\mathbb{R}^{d}\right)}^{2}=\int_{\mathbb{R}^{d}}|\hat{f}(x)|^{2} \cdot v_{s}(x) \mathrm{d} x
$$

Furthermore let $C_{c}\left(\mathbb{R}^{d}\right)$ be the set of all continuous real valued functions with compact support in $\mathbb{R}^{d}$.

We will first present an unbiased Monte Carlo method for integration on $C_{c}\left(\mathbb{R}^{d}\right)$ 
in Section 4. We will examine its error for the subspaces $\stackrel{\circ}{H}^{r, \operatorname{mix}}\left(\mathbb{R}^{d}\right)$ and $\stackrel{\circ}{H}^{s}\left(\mathbb{R}^{d}\right)$ of functions in $H^{r \text { mix }}\left(\mathbb{R}^{d}\right)$ or $H^{s}\left(\mathbb{R}^{d}\right)$ with compact support. This includes an error bound for the classes $\stackrel{\circ}{H}^{r, \operatorname{mix}}\left([0,1]^{d}\right)$ and $\stackrel{\circ}{H}^{s}\left([0,1]^{d}\right)$ of all functions in $H^{r, \operatorname{mix}}\left(\mathbb{R}^{d}\right)$ or $H^{s}\left(\mathbb{R}^{d}\right)$ with support in the unit cube $[0,1]^{d}$. These spaces can also be considered as subspaces of the Hilbert space

$$
H^{r, \operatorname{mix}}\left([0,1]^{d}\right)=\left\{f \in L^{2}\left([0,1]^{d}\right) \mid D^{\alpha} f \in L^{2}\left([0,1]^{d}\right) \text { for every } \alpha \in\{0, \ldots, r\}^{d}\right\}
$$

equipped with the scalar product

$$
\langle f, g\rangle_{H^{r, \operatorname{mix}\left([0,1]^{d}\right)}}=\sum_{\alpha \in\{0, \ldots, r\}^{d}}\left\langle D^{\alpha} f, D^{\alpha} g\right\rangle_{L^{2}\left([0,1]^{d}\right)},
$$

or the Hilbert space

$$
H^{s}\left([0,1]^{d}\right)=\left\{f \in L^{2}\left([0,1]^{d}\right) \mid D^{\alpha} f \in L^{2}\left([0,1]^{d}\right) \text { for } \alpha \in \mathbb{N}_{0}^{d} \text { with }\|\alpha\|_{1} \leq s\right\},
$$

with the scalar product

$$
\langle f, g\rangle_{H^{s}\left([0,1]^{d}\right)}=\sum_{\|\alpha\|_{1} \leq s}\left\langle D^{\alpha} f, D^{\alpha} g\right\rangle_{L^{2}\left([0,1]^{d}\right)}
$$

respectively. It turns out that this method for $\stackrel{\circ}{H}^{r, \operatorname{mix}}\left([0,1]^{d}\right)$ and $\stackrel{\circ}{H}^{s}\left([0,1]^{d}\right)$ can be transformed to the full spaces $H^{r, \operatorname{mix}}\left([0,1]^{d}\right)$ and $H^{s}\left([0,1]^{d}\right)$ without loosing its good properties.

\section{The Basic Quadrature Rule $Q_{S, v}$}

Let $S \in \mathbb{R}^{d \times d}$ be any invertible matrix and $v$ any vector in $\mathbb{R}^{d}$. At the basis of the Monte Carlo methods to be presented is the deterministic and linear quadrature rule $Q_{S, v}$, defined by

$$
Q_{S, v}(f)=\frac{1}{|\operatorname{det} S|} \sum_{m \in \mathbb{Z}^{d}} f\left(S^{-\top}(m+v)\right)
$$

for any admissible input function $f: \mathbb{R}^{d} \rightarrow \mathbb{R}$. This includes all functions $f$ with compact support. For such functions the sum is actually a finite sum. More precisely, $Q_{S, v}$ uses the nodes $S^{-\top}(m+v)$, where $m \in \mathbb{Z}^{d}$ is a lattice point in the 
compact set $\left(S^{\top}(\operatorname{supp} f)-v\right)$ of Lebesgue measure $\left(\operatorname{det}(S) \cdot \lambda_{d}(\operatorname{supp} f)\right)$. This volume is the approximate number of nodes of $Q_{S, v}$.

In particular, the number of nodes of $Q_{a S, v}$ for $a \geq 1$ is of order $a^{d}$. The following simple lemma gives an exact upper bound, see [6] for other bounds.

Lemma 1. Suppose $f: \mathbb{R}^{d} \rightarrow \mathbb{R}$ is supported in an axis-parallel cube of edge length $l>0$. For any invertible matrix $S \in \mathbb{R}^{d \times d}, v \in \mathbb{R}^{d}$ and $a \geq 1$ the quadrature rule $Q_{a S, v}$ uses at most $\left(l \cdot\|S\|_{1}+1\right)^{d} \cdot a^{d}$ function values of $f$.

Proof. By assumption, $f$ has compact support in $\frac{l}{2} \cdot[-1,1]^{d}+x_{0}$ for some $x_{0} \in \mathbb{R}^{d}$. The number of function values is bounded by the size of

$$
\begin{aligned}
M & =\left\{m \in \mathbb{Z}^{d} \mid(a S)^{-\top}(m+v) \in \frac{l}{2} \cdot[-1,1]^{d}+x_{0}\right\} \\
& =\left\{m \in \mathbb{Z}^{d} \mid m+\left(v-a S^{\top} x_{0}\right) \in \frac{a l}{2} \cdot S^{\top}[-1,1]^{d}\right\} .
\end{aligned}
$$

Since $\left\|S^{\top} x\right\|_{\infty} \leq\left\|S^{\top}\right\|_{\infty}=\|S\|_{1}$ for $x \in[-1,1]^{d}$,

$$
M \subseteq\left\{m \in \mathbb{Z}^{d} \mid m+\left(v-a S^{\top} x_{0}\right) \in\left[-\frac{a l}{2}\|S\|_{1}, \frac{a l}{2}\|S\|_{1}\right]^{d}\right\}
$$

and $|M| \leq\left(a l\|S\|_{1}+1\right)^{d}$. With $1 \leq a$ we get the estimate of Lemma 1.

The error of this algorithm for integration on $C_{c}\left(\mathbb{R}^{d}\right)$ can be expressed in terms of the Fourier transform.

Lemma 2. For any invertible matrix $S \in \mathbb{R}^{d \times d}, v \in \mathbb{R}^{d}$ and $f \in C_{c}\left(\mathbb{R}^{d}\right)$

$$
\left|Q_{S, v}(f)-I_{d}(f)\right| \leq \sum_{m \in \mathbb{Z}^{d} \backslash\{0\}}|\hat{f}(S m)|
$$

Proof. The function $g=f \circ S^{-\top}(\cdot+v)$ is continuous with compact support. Hence, 
the Poisson summation formula and an affine linear substitution $x=S^{\top} y-v$ yield

$$
\begin{aligned}
Q_{S, v}(f) & =\frac{1}{|\operatorname{det} S|} \sum_{m \in \mathbb{Z}^{d}} g(m)=\frac{1}{|\operatorname{det} S|} \sum_{m \in \mathbb{Z}^{d}} \hat{g}(m) \\
& =\frac{1}{|\operatorname{det} S|} \sum_{m \in \mathbb{Z}^{d}} \int_{\mathbb{R}^{d}} f\left(S^{-\top}(x+v)\right) \cdot e^{-2 \pi i\langle x, m\rangle} \mathrm{d} x \\
& =\sum_{m \in \mathbb{Z}^{d}} \int_{\mathbb{R}^{d}} f(y) \cdot e^{-2 \pi i\left\langle S^{\top} y-v, m\right\rangle} \mathrm{d} y \\
& =\sum_{m \in \mathbb{Z}^{d}} \hat{f}(S m) \cdot e^{2 \pi i\langle v, m\rangle},
\end{aligned}
$$

if the latter series converges absolutely, see [3, pp. 356]. If not, the stated inequality is obvious. This proves the statement, since $I_{d}(f)=\hat{f}(S \cdot 0) \cdot e^{2 \pi i\langle v, 0\rangle}$.

\section{The Method $M_{a, B}$ for Integration on $\stackrel{\circ}{H}^{r, \operatorname{mix}}\left(\mathbb{R}^{d}\right)$ and $\stackrel{\circ}{H}^{s}\left(\mathbb{R}^{d}\right)$}

It is known how to choose $S$ in $Q_{S, v}$ to get a good deterministic quadrature rule on $\stackrel{\circ}{H}^{r, m i x}\left([0,1]^{d}\right)$. Let the matrix $B \in \mathbb{R}^{d \times d}$ satisfy the following three conditions:

(a) $B$ is invertible,

(b) $\left|\prod_{j=1}^{d}(B m)_{j}\right| \geq 1$, for any $m \in \mathbb{Z}^{d} \backslash\{0\}$,

(c) For any $x, y \in \mathbb{R}^{d}$ the box $[x, y]$ with volume $V=\prod_{j=1}^{d}\left|x_{j}-y_{j}\right|$ contains at most $V+1$ lattice points $B m, m \in \mathbb{Z}^{d}$,

where $[x, y]=\left\{z \in \mathbb{R}^{d} \mid z_{j}\right.$ is inbetween of $x_{j}$ and $y_{j}$ for $\left.j=1, \ldots, d\right\}$. Such a matrix shall be called a Frolov matrix. Property (b) says that for $a>0$ every point of the lattice $a B \mathbb{Z}^{d}$ but zero lies in the set $D_{a}$ of all $x \in \mathbb{R}^{d}$ with $\prod_{j=1}^{d}\left|x_{j}\right| \geq a^{d}$. 


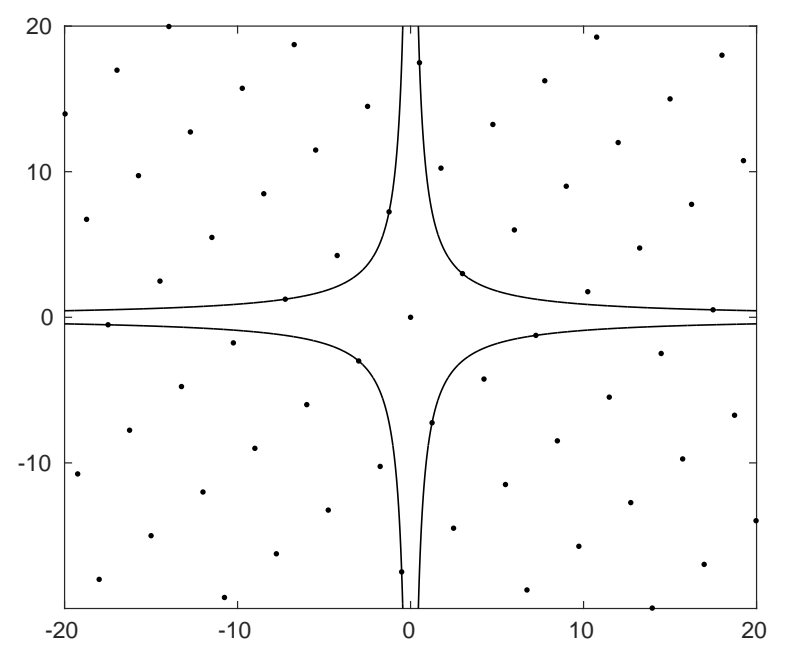

This graphic shows the lattice $a B \mathbb{Z}^{d}$ for $d=2, a=3$ and the Frolov matrix

$$
B=\left(\begin{array}{ll}
1 & 2-\sqrt{2} \\
1 & 2+\sqrt{2}
\end{array}\right) .
$$

Except zero, every lattice point lies inside $D_{3}$.

It is known that one can construct such a matrix $B$ in the following way. Let $p \in \mathbb{Z}[x]$ be a polynomial of degree $d$ with leading coefficient 1 which is irreducible over $\mathbb{Q}$ and has $d$ different real roots $\zeta_{1}, \ldots, \zeta_{d}$. Then the matrix

$$
B=\left(\zeta_{i}^{j-1}\right)_{i, j=1}^{d}
$$

has the desired properties, as shown in [7, p. 364] and [9]. In arbitrary dimension $d$ we can choose $p(x)=(x-1)(x-3) \cdot \ldots \cdot(x-2 d+1)-1$, see [1] or [9], but there are many other possible choices. For example, if $d$ is a power of two, we can set $p(x)=2 \cos (d \cdot \arccos (x / 2))=2 T_{d}(x / 2)$, where $T_{d}$ is the Chebyshev polynomial of degree $d$, see [7, p. 365]. Then the roots of $p$ are explicitly given by $\zeta_{j}=2 \cos \left(\frac{2 j-1}{2 d} \pi\right)$ for $j=1, \ldots, d$.

K. K. Frolov has already seen in 1976 that the algorithm $Q_{a B, 0}$ for $a>1$ is optimal on $\stackrel{\circ}{H}^{r, \operatorname{mix}}\left([0,1]^{d}\right)$ in the sense of order of convergence. It satisfies

$$
\left|Q_{a B, 0}(f)-I_{d}(f)\right| \leq c a^{-r d} \cdot(\log a)^{\frac{d-1}{2}} \cdot\|f\|_{H^{r, \operatorname{mix}\left([0,1]^{d}\right)}}
$$

for a constant $c>0$ and any $a \geq 2$ and $f \in \stackrel{\circ}{H}^{r, \operatorname{mix}}\left([0,1]^{d}\right)$. We hence call it Frolov quadrature formula. See also [1] or [9] for a proof. In fact, the same error bound holds for $Q_{a B, v}$ for any $v \in \mathbb{R}^{d}$.

We define a randomized version of this quadrature rule by introducing two independent random vectors $v$ and $u$. With the random shift parameter $v \in[0,1]^{d}$ the algorithm gets unbiased. The random dilation parameter $u \in\left[1,2^{1 / d}\right]^{d}$ will ensure the general error bound of Theorem 1. Both effects are independent of each other: The random shift is not needed for the error bound and the dilation 
is not needed for the unbiasedness.

Algorithm. For a Frolov matrix $B \in \mathbb{R}^{d \times d}$ and any $a>0$ the randomized Frolov quadrature formula $M_{a, B}$ is the method $Q_{a \bar{u} B, v}$ from Section 3 with independent random vectors $u$ and $v$, uniformly distributed in $\left[1,2^{1 / d}\right]^{d}$ and $[0,1]^{d}$ respectively and $\bar{u}=\operatorname{diag}\left(u_{1}, \ldots, u_{d}\right)$.

Lemma 3. The method $M_{a, B}$ is well-defined and unbiased on $L^{1}\left(\mathbb{R}^{d}\right)$.

Proof. We realize that for $f \in L^{1}\left(\mathbb{R}^{d}\right)$

$$
\begin{aligned}
\mathbb{E}_{u} \mathbb{E}_{v}\left|Q_{a \bar{u} B, v}(f)\right| & \leq \mathbb{E}_{u} \sum_{m \in \mathbb{Z}^{d}} \frac{1}{|\operatorname{det} a \bar{u} B|} \int_{[0,1]^{d}}\left|f\left((a \bar{u} B)^{-\top}(m+x)\right)\right| \mathrm{d} x \\
& =\mathbb{E}_{u} \sum_{m \in \mathbb{Z}^{d}} \int_{(a \bar{u} B)^{-\top} m+(a \bar{u} B)^{-\top}[0,1]^{d}}|f(y)| \mathrm{d} y \\
& =\mathbb{E}_{u} \int_{\mathbb{R}^{d}}|f(y)| \mathrm{d} y=\int_{\mathbb{R}^{d}}|f(y)| \mathrm{d} y<\infty .
\end{aligned}
$$

We can thus apply Fubini's theorem and get

$$
\begin{aligned}
\mathbb{E}\left(M_{a, B}(f)\right) & =\mathbb{E}_{u} \mathbb{E}_{v}\left(Q_{a \bar{u} B, v}(f)\right) \\
& =\mathbb{E}_{u} \sum_{m \in \mathbb{Z}^{d}} \frac{1}{|\operatorname{det} a \bar{u} B|} \int_{[0,1]^{d}} f\left((a \bar{u} B)^{-\top}(m+x)\right) \mathrm{d} x \\
& =\mathbb{E}_{u} \sum_{m \in \mathbb{Z}^{d}} \int_{(a \bar{u} B)^{-\top} m+(a \bar{u} B)^{-\top}[0,1]^{d}} f(y) \mathrm{d} y \\
& =\mathbb{E}_{u} \int_{\mathbb{R}^{d}} f(y) \mathrm{d} y=I_{d}(f) .
\end{aligned}
$$

In particular, $M_{a, B}(f)$ is almost surely finite.

According to Lemma 1 the method $M_{a, B}$ uses no more than $2 \cdot\left(l \cdot\|B\|_{1}+1\right)^{d} \cdot a^{d}$ function values of a function $f$ supported in a cube of edge length $l$. Later we will show that $M_{a, B}$ satisfies

$$
\mathbb{E}\left|M_{a, B}(f)-I_{d}(f)\right| \leq c a^{-r d-d / 2} \cdot(\log a)^{\frac{d-1}{2}} \cdot\|f\|_{H^{r, \operatorname{mix}\left(\mathbb{R}^{d}\right)}}
$$

for a constant $c>0$ and any $a \geq 2^{1 / d}$ and $f \in \stackrel{\circ}{H}^{r, \operatorname{mix}}\left(\mathbb{R}^{d}\right)$. But first we analyze $M_{a, B}$ on the larger set $C_{c}\left(\mathbb{R}^{d}\right)$. 


\section{Error Bound for $C_{c}\left(\mathbb{R}^{d}\right)$}

We prove a main result of this paper. Again, $D_{a}$ is the set of all $x \in \mathbb{R}^{d}$ with $\prod_{j=1}^{d}\left|x_{j}\right| \geq a^{d}$. The method $M_{a, B}$ satisfies a general error bound on $C_{c}\left(\mathbb{R}^{d}\right)$.

Theorem 1. Let $B \in \mathbb{R}^{d \times d}$ be a Frolov matrix. Then there is a constant $c>0$ such that for every $a>0$ and $f \in C_{c}\left(\mathbb{R}^{d}\right)$

$$
\mathbb{E}\left|M_{a, B}(f)-I_{d}(f)\right| \leq c a^{-d} \cdot \int_{D_{a}}|\hat{f}(x)| \mathrm{d} x .
$$

Proof. Let $v \in \mathbb{R}^{d}$ be arbitrary, but fixed. Thanks to Lemma 2 and the monotone convergence theorem we have

$$
\mathbb{E}_{u}\left|Q_{a \bar{u} B, v}(f)-I_{d}(f)\right| \leq \mathbb{E}_{u}\left(\sum_{m \in \mathbb{Z}^{d} \backslash\{0\}}|\hat{f}(a \bar{u} B m)|\right)=\sum_{m \in \mathbb{Z}^{d} \backslash\{0\}} \mathbb{E}_{u}|\hat{f}(a \bar{u} B m)|
$$

Since each $a \bar{u} B m$ is uniformly distributed in the box $\left[a B m, 2^{1 / d} a B m\right]$ with volume $\left(2^{1 / d}-1\right)^{d} \cdot\left|\prod_{j=1}^{d} a(B m)_{j}\right|$, this series equals

$$
\begin{aligned}
& \frac{1}{\left(2^{1 / d}-1\right)^{d}} \sum_{m \in \mathbb{Z}^{d} \backslash\{0\}} \int_{\left[a B m, 2^{1 / d} a B m\right]} \frac{|\hat{f}(x)|}{\prod_{j=1}^{d}\left|a(B m)_{j}\right|} \mathrm{d} x \\
& \leq \frac{1}{\left(2^{1 / d}-1\right)^{d}} \sum_{m \in \mathbb{Z}^{d} \backslash\{0\}} \int_{\left[a B m, 2^{1 / d} a B m\right]} \frac{|\hat{f}(x)|}{\prod_{j=1}^{d} 2^{-1 / d}\left|x_{j}\right|} \mathrm{d} x \\
& =\frac{2}{\left(2^{1 / d}-1\right)^{d}} \cdot \int_{\mathbb{R}^{d}} \frac{|\hat{f}(x)|}{\prod_{j=1}^{d}\left|x_{j}\right|} \cdot\left|\left\{m \in \mathbb{Z}^{d} \backslash\{0\} \mid x \in\left[a B m, 2^{1 / d} a B m\right]\right\}\right| \mathrm{d} x \\
& =\frac{2}{\left(2^{1 / d}-1\right)^{d}} \cdot \int_{\mathbb{R}^{d}} \frac{|\hat{f}(x)|}{\prod_{j=1}^{d}\left|x_{j}\right|} \cdot\left|\left\{m \in \mathbb{Z}^{d} \backslash\{0\} \mid B m \in\left[\frac{x}{2^{1 / d} a}, \frac{x}{a}\right]\right\}\right| \mathrm{d} x .
\end{aligned}
$$

Thanks to the properties of the Frolov matrix $B$, if $\prod_{j=1}^{d}\left|x_{j}\right|<a^{d}$, the latter set is empty and otherwise contains no more than $\prod_{j=1}^{d}\left|\frac{x_{j}}{a}\right|+1 \leq 2 a^{-d} \prod_{j=1}^{d}\left|x_{j}\right|$ points. Thus, we arrive at

$$
\mathbb{E}_{u}\left|Q_{a \bar{u} B, v}(f)-I_{d}(f)\right| \leq \frac{4}{\left(2^{1 / d}-1\right)^{d}} \cdot a^{-d} \int_{D_{a}}|\hat{f}(x)| \mathrm{d} x .
$$


By Fubini's theorem, we have

$$
\mathbb{E}\left|M_{a, B}(f)-I_{d}(f)\right|=\mathbb{E}_{v} \mathbb{E}_{u}\left|Q_{a \bar{u} B, v}(f)-I_{d}(f)\right| \leq \frac{4}{\left(2^{1 / d}-1\right)^{d}} \cdot a^{-d} \int_{D_{a}}|\hat{f}(x)| \mathrm{d} x
$$

and the theorem is proven.

Additional differentiability properties of $f \in C_{c}\left(\mathbb{R}^{d}\right)$ result in decay properties of $\hat{f}$. This leads to estimates of the integral $\int_{D_{a}}|\hat{f}(x)| \mathrm{d} x$. Hence, the general upper bound for the error of $M_{a, B}(f)$ in Theorem 1 adjusts to the differentiability of $f$. Two such examples are functions from $\stackrel{\circ}{H}^{r, \operatorname{mix}}\left(\mathbb{R}^{d}\right)$ and $\stackrel{\circ}{H}^{s}\left(\mathbb{R}^{d}\right)$.

\section{Error Bounds for $\stackrel{\circ}{H}^{r, \operatorname{mix}}\left(\mathbb{R}^{d}\right)$}

If $f \in \stackrel{\circ}{H}^{r, \operatorname{mix}}\left(\mathbb{R}^{d}\right) \subseteq C_{c}\left(\mathbb{R}^{d}\right)$, the following lemma holds.

Lemma 4. For any Frolov matrix $B \in \mathbb{R}^{d \times d}$ and $r \in \mathbb{N}$ there is some $c>0$ such that for each $a \geq 2^{1 / d}$ and $f \in \stackrel{\circ}{H}^{r, \operatorname{mix}}\left(\mathbb{R}^{d}\right)$

$$
\int_{D_{a}}|\hat{f}(x)| \mathrm{d} x \leq c a^{-r d+d / 2}(\log a)^{\frac{d-1}{2}}\|f\|_{H^{r, \operatorname{mix}\left(\mathbb{R}^{d}\right)}} .
$$

Proof. Applying Hölder's inequality and a linear substitution $x=a B y$ to the above integral, we get

$$
\begin{aligned}
& \left(\int_{D_{a}}|\hat{f}(x)| \mathrm{d} x\right)^{2}=\left(\int_{D_{a}}|\hat{f}(x)| h_{r}(x)^{1 / 2} \cdot h_{r}(x)^{-1 / 2} \mathrm{~d} x\right)^{2} \\
& \leq\|f\|_{H^{r, \text { mix }\left(\mathbb{R}^{d}\right)}}^{2} \cdot \int_{D_{a}} h_{r}(x)^{-1} \mathrm{~d} x=\|f\|_{H^{r, \text { mix }\left(\mathbb{R}^{d}\right)}}^{2} \cdot\left(\int_{G} h_{r}(a B y)^{-1} \mathrm{~d} y\right) \cdot a^{d}|\operatorname{det} B|,
\end{aligned}
$$

where $G=B^{-1} D_{1}$ is the set of all $y \in \mathbb{R}^{d}$ with $\prod_{j=1}^{d}\left|(B y)_{j}\right| \geq 1$. It it thus sufficient to prove that the integral $\int_{G} h_{r}(a B y)^{-1} \mathrm{~d} y$ is bounded by a constant multiple of $a^{-2 r d} \cdot(\log a)^{d-1}$.

Consider the auxiliary set $N(\beta)=\left\{x \in \mathbb{R}^{d}\left|\left\lfloor 2^{\beta_{j}-1}\right\rfloor \leq\right| x_{j} \mid<2^{\beta_{j}}, 1 \leq j \leq d\right\}$ for $\beta \in \mathbb{N}_{0}^{d}$. Let $|\beta|=\sum_{j=1}^{d} \beta_{j}$ and $G_{a}^{\beta}=G \cap\left\{y \in \mathbb{R}^{d} \mid a B y \in N(\beta)\right\}$. Since $\mathbb{R}^{d}$ is the disjoint union of all $N(\beta), G$ is the disjoint union of all $G_{a}^{\beta}$ over $\beta \in \mathbb{N}_{0}^{d}$. For $y \in G_{a}^{\beta}$ we have both $\left|\prod_{j=1}^{d} a(B y)_{j}\right| \geq a^{d}$, since $y \in G$, and $\left|\prod_{j=1}^{d} a(B y)_{j}\right|<2^{|\beta|}$, since $a B y \in N(\beta)$. This implies $G_{a}^{\beta}=\emptyset$ for $|\beta| \leq d \log _{2} a$, since then $2^{|\beta|} \leq a^{d}$. 
Let $y \in G_{a}^{\beta}$ and $|\beta|>d \log _{2} a$. Then

$$
h_{r}(a B y) \geq \prod_{j=1}^{d}\left(1+\left\lfloor 2^{\beta_{j}-1}\right\rfloor^{2 r}\right) \geq \prod_{j=1}^{d} 2^{2 r\left(\beta_{j}-1\right)}=2^{2 r(|\beta|-d)}
$$

and hence $h_{r}(a B y)^{-1} \leq 2^{2 r(d-|\beta|)}$. On the other hand

$$
\begin{aligned}
& \lambda_{d}\left(G_{a}^{\beta}\right) \leq \lambda_{d}\left((a B)^{-1} N(\beta)\right)=a^{-d} \cdot|\operatorname{det} B|^{-1} \cdot \lambda_{d}(N(\beta)) \\
& =a^{-d} \cdot|\operatorname{det} B|^{-1} \cdot 2^{d} \cdot \prod_{j=1}^{d}\left(2^{\beta_{j}}-\left\lfloor 2^{\beta_{j}-1}\right\rfloor\right) \leq a^{-d} \cdot|\operatorname{det} B|^{-1} \cdot 2^{d} \cdot 2^{|\beta|} .
\end{aligned}
$$

Together we obtain

$$
\begin{aligned}
& \int_{G} h_{r}(a B y)^{-1} \mathrm{~d} y=\sum_{\beta \in \mathbb{N}_{0}^{d}} \int_{G_{a}^{\beta}} h_{r}(a B y)^{-1} \mathrm{~d} y \\
& =\sum_{|\beta|>d \log _{2} a} \int_{G_{a}^{\beta}} h_{r}(a B y)^{-1} \mathrm{~d} y \\
& \leq \sum_{|\beta|>d \log _{2} a} 2^{2 r(d-|\beta|)} \cdot a^{-d} \cdot|\operatorname{det} B|^{-1} \cdot 2^{d} \cdot 2^{|\beta|} \\
& \leq 2^{2 r d+d}|\operatorname{det} B|^{-1} \cdot a^{-d} \sum_{k=\left\lceil d \log _{2} a\right\rceil}^{\infty} 2^{(1-2 r) k} \cdot\left|\left\{\beta \in \mathbb{N}_{0}^{d}|| \beta \mid=k\right\}\right| \\
& \leq 2^{2 r d+d}|\operatorname{det} B|^{-1} \cdot a^{-d} \sum_{k=\left\lceil d \log _{2} a\right\rceil}^{\infty} 2^{(1-2 r) k} \cdot(k+1)^{d-1} \\
& =2^{2 r d+d}|\operatorname{det} B|^{-1} \cdot a^{-d} \sum_{k=0}^{\infty} 2^{(1-2 r)\left(k+\left\lceil d \log _{2} a\right\rceil\right)} \cdot\left(k+1+\left\lceil d \log _{2} a\right\rceil\right)^{d-1} \\
& \leq 2^{2 r d+d}|\operatorname{det} B|^{-1} \cdot a^{-d} \cdot a^{(1-2 r) d} \cdot \sum_{k=0}^{\infty} 2^{(1-2 r) k} \cdot 2^{d-1} \cdot(k+1)^{d-1} \cdot\left\lceil d \log _{2} a\right\rceil^{d-1} \\
& d \log _{2} a \geq 1 \\
& \leq \\
& \leq \\
& 2^{2 r d+2 d-1}|\operatorname{det} B|^{-1} \cdot a^{-2 r d} \cdot \sum_{k=0}^{\infty} 2^{(1-2 r) k}(k+1)^{d-1}\left(2 d \cdot \frac{\log a}{\log 2}\right)^{d-1} \\
& =\left(2^{2 r d+3 d-2} d^{d-1}|\operatorname{det} B|^{-1}\left(\log ^{2 r} 2\right)^{1-d} \sum_{k=0}^{\infty}\left(2^{1-2 r}\right)^{k}(k+1)^{d-1}\right) \cdot a^{-2 r d} \cdot\left(\log ^{2} a\right)^{d-1} .
\end{aligned}
$$

This is the desired estimate, since $2^{1-2 r}<1$.

Combining Theorem 1 and Lemma 4 yields: 
Theorem 2. Let $B \in \mathbb{R}^{d \times d}$ be a Frolov matrix and $r \in \mathbb{N}$. Then there is a constant $c>0$ such that for every $a \geq 2^{1 / d}$ and $f \in \stackrel{\circ}{H}^{r, \operatorname{mix}}\left(\mathbb{R}^{d}\right)$

$$
\mathbb{E}\left|M_{a, B}(f)-I_{d}(f)\right| \leq c a^{-r d-d / 2}(\log a)^{\frac{d-1}{2}}\|f\|_{H^{r, \operatorname{mix}\left(\mathbb{R}^{d}\right)}} .
$$

The worst case error of $M_{a, B}$ for functions in $\stackrel{\circ}{H}^{r, \operatorname{mix}}\left([0,1]^{d}\right)$ is small, too.

Theorem 3. Let $B \in \mathbb{R}^{d \times d}$ be a Frolov matrix and $r \in \mathbb{N}$. Then there is a constant $c>0$ such that for every $a \geq 2^{1 / d}$ and $f \in \stackrel{\circ}{H}^{r, \operatorname{mix}}\left([0,1]^{d}\right)$

$$
\sup _{\omega}\left|M_{a, B}^{\omega}(f)-I_{d}(f)\right| \leq c a^{-r d}(\log a)^{\frac{d-1}{2}}\|f\|_{H^{r, \operatorname{mix}\left([0,1]^{d}\right)}}
$$

where the supremum is taken over all realizations $M_{a, B}^{\omega}$ of $M_{a, B}$.

Proof. The realizations $M_{a, B}^{\omega}$ of $M_{a, B}$ take the form $Q_{a \bar{u} B, v}$ for some $u \in\left[1,2^{1 / d}\right]^{d}$ and $v \in[0,1]^{d}$. By Lemma 2 and Hölder's inequality,

$$
\begin{aligned}
& \left|Q_{a \bar{u} B, v}(f)-I_{d}(f)\right|^{2} \leq\left(\sum_{m \in \mathbb{Z}^{d} \backslash\{0\}}|\hat{f}(a \bar{u} B m)|\right)^{2} \\
& \leq\left(\sum_{m \in \mathbb{Z}^{d} \backslash\{0\}} h_{r}(a \bar{u} B m)^{-1}\right) \cdot\left(\sum_{m \in \mathbb{Z}^{d} \backslash\{0\}} h_{r}(a \bar{u} B m) \cdot|\hat{f}(a \bar{u} B m)|^{2}\right) .
\end{aligned}
$$

The first factor of this product is bounded above by a constant multiple of $a^{-2 r d}$. $(\log a)^{d-1}$. This is proven similar to Lemma 4.

Let $N(\beta)=\left\{x \in \mathbb{R}^{d}\left|\left\lfloor 2^{\beta_{j}-1}\right\rfloor \leq\right| x_{j} \mid<2^{\beta_{j}}, 1 \leq j \leq d\right\}$ for $\beta \in \mathbb{N}_{0}^{d}$ and $G_{a}^{\beta}=\left\{m \in \mathbb{Z}^{d} \backslash\{0\} \mid a \bar{u} B m \in N(\beta)\right\}$. Then $\mathbb{Z}^{d} \backslash\{0\}$ is the disjoint union of all $G_{a}^{\beta}$ over $\beta \in \mathbb{N}_{0}^{d}$. Again $G_{a}^{\beta}$ is empty for $|\beta| \leq d \log _{2} a$. Otherwise,

$$
h_{r}(a \bar{u} B m) \geq \prod_{j=1}^{d}\left(1+\left\lfloor 2^{\beta_{j}-1}\right\rfloor^{2 r}\right) \geq \prod_{j=1}^{d} 2^{2 r\left(\beta_{j}-1\right)}=2^{2 r(|\beta|-d)}
$$

for $m \in G_{a}^{\beta}$ and hence $h_{r}(a \bar{u} B m)^{-1} \leq 2^{2 r(d-|\beta|)}$, and

$$
\left|G_{a}^{\beta}\right| \leq\left|\left\{m \in \mathbb{Z}^{d} \backslash\{0\}||(B m)_{j} \mid<\frac{2^{\beta_{j}}}{a}\right\}\right| \leq 2^{d+|\beta|} a^{-d}+1 \leq 2^{d+1+|\beta|} a^{-d},
$$


since $B$ is a Frolov matrix. This yields

$$
\begin{aligned}
\sum_{m \in \mathbb{Z}^{d} \backslash\{0\}} h_{r}(a \bar{u} B m)^{-1} & =\sum_{\beta \in \mathbb{N}_{0}^{d}} \sum_{m \in G_{a}^{\beta}} h_{r}(a \bar{u} B m)^{-1} \leq \sum_{|\beta|>d \log _{2} a} 2^{2 r(d-|\beta|)} \cdot a^{-d} \cdot 2^{d+1+|\beta|} \\
& \leq c_{1} \cdot a^{-2 r d} \cdot(\log a)^{d-1},
\end{aligned}
$$

like in Lemma 4.

We show that the second factor in the above inequality is bounded above by a

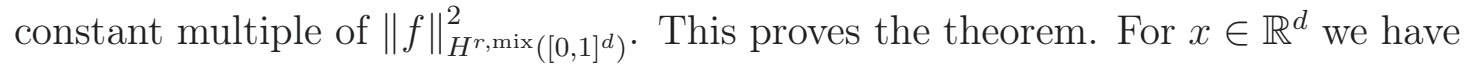

$$
h_{r}(x) \cdot|\hat{f}(x)|^{2}=\sum_{\alpha \in\{0, \ldots, r\}^{d}}\left|\widehat{D^{\alpha} f}(x)\right|^{2} .
$$

The function $g_{\alpha}=D^{\alpha} f \circ(a \bar{u} B)^{-\top}$ has compact support in $(a \bar{u} B)^{\top}[0,1]^{d}$. Let $M_{a}=\left\{k \in \mathbb{Z}^{d} \mid k+[0,1]^{d} \cap(a \bar{u} B)^{\top}[0,1]^{d} \neq \emptyset\right\}$. Then

$$
\begin{aligned}
\left|\widehat{D^{\alpha} f}(a \bar{u} B m)\right|^{2} & =\left|\int_{\mathbb{R}^{d}} D^{\alpha} f(y) \cdot e^{-2 \pi i\langle a \bar{u} B m, y\rangle} \mathrm{d} y\right|^{2} \\
& =\left|\frac{1}{\operatorname{det}(a \bar{u} B)} \int_{\mathbb{R}^{d}} g_{\alpha}(x) \cdot e^{-2 \pi i\langle m, x\rangle} \mathrm{d} x\right|^{2} \\
& =\left.\left|\frac{1}{\operatorname{det}(a \bar{u} B)} \sum_{k \in M_{a}}\left\langle g_{\alpha}(x), e^{2 \pi i\langle m, \cdot\rangle}\right\rangle_{L^{2}(k+[0,1]}\right|^{2}\right|^{2} \\
& \leq \frac{\left|M_{a}\right|}{|\operatorname{det}(a \bar{u} B)|^{2}} \sum_{k \in M_{a}}\left|\left\langle g_{\alpha}, e^{2 \pi i\langle m, \cdot\rangle}\right\rangle_{L^{2}(k+[0,1] d)}\right|^{2} .
\end{aligned}
$$

Thus we obtain

$$
\begin{aligned}
& \sum_{m \in \mathbb{Z}^{d} \backslash\{0\}} h_{r}(a \bar{u} B m) \cdot|\hat{f}(a \bar{u} B m)|^{2} \leq \sum_{m \in \mathbb{Z}^{d}} \sum_{\alpha \in\{0, \ldots, r\}^{d}}\left|\widehat{D^{\alpha} f}(a \bar{u} B m)\right|^{2} \\
\leq & \frac{\left|M_{a}\right|}{|\operatorname{det}(a \bar{u} B)|^{2}} \sum_{m \in \mathbb{Z}^{d}} \sum_{\alpha \in\{0, \ldots, r\}^{d}} \sum_{k \in M_{a}}\left|\left\langle g_{\alpha}, e^{2 \pi i\langle m, \cdot\rangle}\right\rangle_{L^{2}\left(k+[0,1]^{d}\right)}\right|^{2} \\
= & \frac{\left|M_{a}\right|}{|\operatorname{det}(a \bar{u} B)|^{2}} \sum_{\alpha \in\{0, \ldots, r\}^{d}} \sum_{k \in M_{a}}\left\|\left.g_{\alpha}\right|_{L^{2}\left(k+[0,1]^{d}\right)} ^{2}=\frac{\left|M_{a}\right|}{|\operatorname{det}(a \bar{u} B)|^{2}} \sum_{\alpha \in\{0, \ldots, r\}^{d}}\right\| g_{\alpha} \|_{L^{2}\left(\mathbb{R}^{d}\right)}^{2} \\
= & \frac{\left|M_{a}\right|}{|\operatorname{det}(a \bar{u} B)|} \sum_{\alpha \in\{0, \ldots, r\}^{d}}\left\|D^{\alpha} f\right\|_{L^{2}\left(\mathbb{R}^{d}\right)}^{2}=\frac{\left|M_{a}\right|}{|\operatorname{det}(a \bar{u} B)|}\|f\|_{H^{r, \text { mix }\left([0,1]^{d}\right)}{ }^{2} .}
\end{aligned}
$$

Since both $\left|M_{a}\right|$ and $|\operatorname{det}(a \bar{u} B)|$ are of order $a^{d}$, this yields the statement. 


\section{Error Bounds for $\stackrel{\circ}{H}^{s}\left(\mathbb{R}^{d}\right)$}

If, however, $s \in \mathbb{N}$ with $s>d / 2$ and the integrand is from $\stackrel{\circ}{H}^{s}\left(\mathbb{R}^{d}\right) \subseteq C_{c}\left(\mathbb{R}^{d}\right)$, the following lemma holds.

Lemma 5. For any Frolov matrix $B \in \mathbb{R}^{d \times d}$ and $s \in \mathbb{N}$ with $s>d / 2$ there is some $c>0$ such that for each $a>0$ and $f \in \stackrel{\circ}{H}^{s}\left(\mathbb{R}^{d}\right)$

$$
\int_{D_{a}}|\hat{f}(x)| \mathrm{d} x \leq c a^{-s+d / 2}\|f\|_{H^{s}\left(\mathbb{R}^{d}\right)} .
$$

Proof. Like in Lemma 4, we apply Hölder's inequality and get

$$
\begin{aligned}
& \left(\int_{D_{a}}|\hat{f}(x)| \mathrm{d} x\right)^{2}=\left(\int_{D_{a}}|\hat{f}(x)| v_{s}(x)^{1 / 2} \cdot v_{s}(x)^{-1 / 2} \mathrm{~d} x\right)^{2} \\
& \leq\left(\int_{D_{a}} v_{s}(x)^{-1} \mathrm{~d} x\right) \cdot\|f\|_{H^{s}\left(\mathbb{R}^{d}\right)}^{2} \leq \tilde{c} \cdot\left(\int_{D_{a}}\left(1+\|x\|_{2}^{2}\right)^{-s} \mathrm{~d} x\right) \cdot\|f\|_{H^{s}\left(\mathbb{R}^{d}\right)}^{2},
\end{aligned}
$$

for some $\tilde{c}>0$. Since $\|x\|_{2} \geq a$ for $x \in D_{a}$, the latter integral is bounded by

$$
\begin{aligned}
& \int_{\left\{x \in \mathbb{R}^{d:}:\|x\|_{2} \geq a\right\}}\left(1+\|x\|_{2}^{2}\right)^{-s} \mathrm{~d} x=\int_{a}^{\infty} \int_{S_{d-1}}\left(1+R^{2}\right)^{-s} \cdot R^{d-1} \mathrm{~d} \sigma \mathrm{d} R \\
= & \sigma\left(S_{d-1}\right) \int_{a}^{\infty}\left(1+R^{2}\right)^{-s} \cdot R^{d-1} \mathrm{~d} R \leq \sigma\left(S_{d-1}\right) \int_{a}^{\infty} R^{-2 s+d-1} \mathrm{~d} R \leq \bar{c} \cdot a^{-2 s+d},
\end{aligned}
$$

for some $\bar{c}>0$, since $-2 s+d-1<-1$.

In this case, combining Theorem 1 and Lemma 5 yields:

Theorem 4. Let $B \in \mathbb{R}^{d \times d}$ be a Frolov matrix, $s \in \mathbb{N}$ and $s>d / 2$. Then there is a constant $c>0$ such that for every $a>0$ and $f \in \stackrel{\circ}{H}^{s}\left(\mathbb{R}^{d}\right)$

$$
\mathbb{E}\left|M_{a, B}(f)-I_{d}(f)\right| \leq c a^{-s-d / 2}\|f\|_{H^{s}\left(\mathbb{R}^{d}\right)} .
$$

The Frolov property of $B$ is important for Theorem 1 and the class $\stackrel{\circ}{H}^{r, \operatorname{mix}}\left([0,1]^{d}\right)$, but we remark that $B$ does not have to be a Frolov matrix to get this estimate on $\stackrel{\circ}{H}^{s}\left(\mathbb{R}^{d}\right)$. As seen in the proof of Lemma 5 , we do not need that the lattice points of $a B \mathbb{Z}^{d} \backslash\{0\}$ lie in $D_{a}$ but only that they lie outside the ball $\left\{x \in \mathbb{R}^{d} \mid\|x\|_{2} \leq a\right\}$. For example, the identity matrix would do. But if $B$ is a Frolov matrix, $M_{a, B}$ works universally for $\stackrel{\circ}{H}^{r, m i x}\left(\mathbb{R}^{d}\right)$ and $\stackrel{\circ}{H}^{s}\left(\mathbb{R}^{d}\right)$. Furthermore, the Frolov properties 
of $B$ prevent extremely large jumps of the number of nodes of $M_{a, B}=Q_{a \bar{u} B, v}$ for small changes of $a>0$ or $u \in\left[1,2^{1 / d}\right]^{d}$.

For functions from $\stackrel{\circ}{H}^{s}\left([0,1]^{d}\right)$ the worst case error of $M_{a, B}$ is also small.

Theorem 5. Let $B \in \mathbb{R}^{d \times d}$ be a Frolov matrix and $s \in \mathbb{N}$ with $s>d / 2$. Then there is a constant $c>0$ such that for every $a>0$ and $f \in \stackrel{\circ}{H}^{s}\left([0,1]^{d}\right)$

$$
\sup _{\omega}\left|M_{a, B}^{\omega}(f)-I_{d}(f)\right| \leq c a^{-s}\|f\|_{H^{s}\left([0,1]^{d}\right)},
$$

where the supremum is taken over all realizations $M_{a, B}^{\omega}$ of $M_{a, B}$.

Proof. The realizations $M_{a, B}^{\omega}$ of $M_{a, B}$ take the form $Q_{a \bar{u} B, v}$ for some $u \in\left[1,2^{1 / d}\right]^{d}$ and $v \in[0,1]^{d}$. By Lemma 2 and Hölder's inequality,

$$
\begin{aligned}
& \left|Q_{a \bar{u} B, v}(f)-I_{d}(f)\right|^{2} \leq\left(\sum_{m \in \mathbb{Z}^{d} \backslash\{0\}}|\hat{f}(a \bar{u} B m)|\right)^{2} \\
& \leq\left(\sum_{m \in \mathbb{Z}^{d} \backslash\{0\}} v_{s}(a \bar{u} B m)^{-1}\right) \cdot\left(\sum_{m \in \mathbb{Z}^{d} \backslash\{0\}} v_{s}(a \bar{u} B m) \cdot|\hat{f}(a \bar{u} B m)|^{2}\right) .
\end{aligned}
$$

The first factor of this product is bounded above by a constant multiple of $a^{-2 s}$ : Since

$$
v_{s}(a \bar{u} B m) \geq\|a \bar{u} B m\|_{2}^{2 s} \geq a^{2 s} \cdot\|B m\|_{2}^{2 s} \geq a^{2 s} \cdot\left\|B^{-1}\right\|_{2}^{-2 s} \cdot\|m\|_{2}^{2 s},
$$

we have

$$
\sum_{m \in \mathbb{Z}^{d} \backslash\{0\}} v_{s}(a \bar{u} B m)^{-1} \leq a^{-2 s} \cdot\left\|B^{-1}\right\|_{2}^{2 s} \cdot \sum_{m \in \mathbb{Z}^{d} \backslash\{0\}}\|m\|_{2}^{-2 s}
$$

where this last series converges for $2 s>d$.

We show that the second factor in the above inequality is bounded above by a constant multiple of $\|f\|_{H^{s}\left([0,1]^{d}\right)}^{2}$. This proves the theorem.

For any $x \in \mathbb{R}^{d}$ we have

$$
v_{s}(x) \cdot|\hat{f}(x)|^{2}=\sum_{\|\alpha\|_{1} \leq s}\left|\widehat{D^{\alpha} f}(x)\right|^{2}
$$

The function $g_{\alpha}=D^{\alpha} f \circ(a \bar{u} B)^{-\top}$ has compact support in $(a \bar{u} B)^{\top}[0,1]^{d}$. Let 


$$
\begin{aligned}
& M_{a}=\left\{k \in \mathbb{Z}^{d} \mid k+[0,1]^{d} \cap(a \bar{u} B)^{\top}[0,1]^{d} \neq \emptyset\right\} . \text { Then } \\
& \left|\widehat{D^{\alpha}} f(a \bar{u} B m)\right|^{2}=\left|\int_{\mathbb{R}^{d}} D^{\alpha} f(y) \cdot e^{-2 \pi i\langle a \bar{u} B m, y\rangle} \mathrm{d} y\right|^{2} \\
& =\left|\frac{1}{\operatorname{det}(a \bar{u} B)} \int_{\mathbb{R}^{d}} g_{\alpha}(x) \cdot e^{-2 \pi i\langle m, x\rangle} \mathrm{d} x\right|^{2} \\
& =\left|\frac{1}{\operatorname{det}(a \bar{u} B)} \sum_{k \in M_{a}}\left\langle g_{\alpha}(x), e^{2 \pi i\langle m, \cdot\rangle}\right\rangle_{L^{2}\left(k+[0,1]^{d}\right)}\right|^{2} \\
& \leq \frac{\left|M_{a}\right|}{|\operatorname{det}(a \bar{u} B)|^{2}} \sum_{k \in M_{a}}\left|\left\langle g_{\alpha}, e^{2 \pi i\langle m, \cdot\rangle}\right\rangle_{L^{2}\left(k+[0,1]^{d}\right)}\right|^{2} \text {. }
\end{aligned}
$$

Thus we obtain

$$
\begin{aligned}
& \sum_{m \in \mathbb{Z}^{d} \backslash\{0\}} v_{s}(a \bar{u} B m) \cdot|\hat{f}(a \bar{u} B m)|^{2} \leq \sum_{m \in \mathbb{Z}^{d}} \sum_{\|\alpha\|_{1} \leq s}\left|\widehat{D^{\alpha}} f(a \bar{u} B m)\right|^{2} \\
\leq & \left.\frac{\left|M_{a}\right|}{|\operatorname{det}(a \bar{u} B)|^{2}} \sum_{m \in \mathbb{Z}^{d}} \sum_{\|\alpha\|_{1} \leq s} \sum_{k \in M_{a}} \mid\left\langle g_{\alpha}, e^{2 \pi i\langle m, \cdot\rangle}\right\rangle_{L^{2}(k+[0,1]}\right)\left.\right|^{2} \\
= & \frac{\left|M_{a}\right|}{|\operatorname{det}(a \bar{u} B)|^{2}} \sum_{\|\alpha\|_{1} \leq s} \sum_{k \in M_{a}}\left\|\left.g_{\alpha}\right|_{L^{2}\left(k+[0,1]^{d}\right)} ^{2}=\frac{\left|M_{a}\right|}{|\operatorname{det}(a \bar{u} B)|^{2}} \sum_{\|\alpha\|_{1} \leq s}\right\| g_{\alpha} \|_{L^{2}\left(\mathbb{R}^{d}\right)}^{2} \\
= & \frac{\left|M_{a}\right|}{|\operatorname{det}(a \bar{u} B)|} \sum_{\|\alpha\|_{1} \leq s}\left\|D^{\alpha} f\right\|_{L^{2}\left(\mathbb{R}^{d}\right)}^{2}=\frac{\left|M_{a}\right|}{|\operatorname{det}(a \bar{u} B)|}\|f\|_{H^{s}\left([0,1]^{d}\right)}{ }^{2} .
\end{aligned}
$$

Since both $\left|M_{a}\right|$ and $|\operatorname{det}(a \bar{u} B)|$ are of order $a^{d}$, this yields the statement.

\section{The Method $\widetilde{M}_{a, B}$ for Integration on $H^{s}\left([0,1]^{d}\right)$ and $H^{r, \operatorname{mix}}\left([0,1]^{d}\right)$}

We can transform the Monte Carlo method $M_{a, B}$ from above such that it is still unbiased and its error satisfies the same upper bounds for the full spaces $H^{r \text {,mix }}\left([0,1]^{d}\right)$ and $H^{s}\left([0,1]^{d}\right)$, that $M_{a, B}$ satisfies for the subspaces $\stackrel{\circ}{H}^{r, \operatorname{mix}}\left([0,1]^{d}\right)$ and $\stackrel{\circ}{H}^{s}\left([0,1]^{d}\right)$. This is done by a standard method, which is also used for deterministic quadrature rules for $\stackrel{\circ}{H}^{r, \operatorname{mix}}\left([0,1]^{d}\right)$, see [8, pp. 359].

To that end let $\psi: \mathbb{R} \rightarrow \mathbb{R}$ be an infinitely differentiable function such that $\left.\psi\right|_{(-\infty, 0)}=0,\left.\psi\right|_{(1, \infty)}=1$ and $\left.\psi\right|_{(0,1)}:(0,1) \rightarrow(0,1)$ is a diffeomorphism. For 
example, we can choose

$$
h(x)=\left\{\begin{array}{ll}
e^{\frac{1}{(2 x-1)^{2}-1}} & \text { if } x \in(0,1), \\
0 & \text { else, }
\end{array} \quad \psi(x)=\frac{\int_{-\infty}^{x} h(t) \mathrm{d} t}{\int_{-\infty}^{\infty} h(t) \mathrm{d} t}\right.
$$

for $x \in \mathbb{R}$. Like $h$ also $\psi$ is infinitely differentiable and obviously satisfies $\left.\psi\right|_{(-\infty, 0)}=$ 0 and $\left.\psi\right|_{(1, \infty)}=1$. Since the derivative of $\psi$ is strictly positive on $(0,1)$, it is strictly increasing and a bijection of $(0,1)$ with a smooth inverse function.
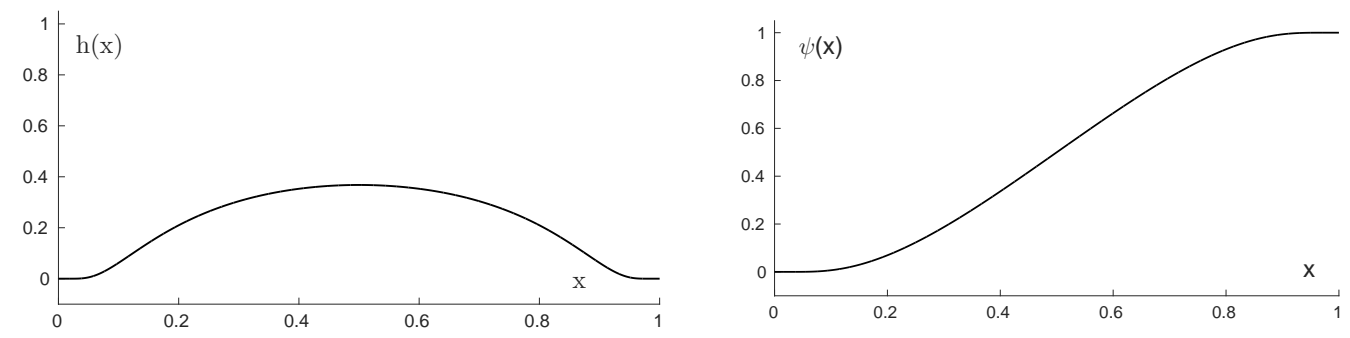

Given such $\psi$, the map $\Psi: \mathbb{R}^{d} \rightarrow \mathbb{R}^{d}$ with $\Psi(x)=\left(\psi\left(x_{1}\right), \ldots, \psi\left(x_{d}\right)\right)^{\top}$ is a diffeomorphism on $(0,1)^{d}$ with inverse $\Psi^{-1}(x)=\left(\psi^{-1}\left(x_{1}\right), \ldots, \psi^{-1}\left(x_{d}\right)\right)^{\top}$ and $|D \Psi(x)| \stackrel{\psi^{\prime} \geq 0}{=} \operatorname{det} D \Psi(x)=\prod_{i=1}^{d} \psi^{\prime}\left(x_{i}\right)$.

If $Q$ is a linear quadrature formula for integration on the unit cube with nodes $x^{(j)} \in[0,1]^{d}$ and weights $a_{j} \in \mathbb{R}$, where $j=1, \ldots, n$, we define the transformed quadrature formula $\widetilde{Q}$ by choosing the nodes and weights to be

$$
\tilde{x}^{(j)}=\Psi\left(x^{(j)}\right) \quad \text { and } \quad \tilde{a_{j}}=a_{j} \cdot\left|D \Psi\left(x^{(j)}\right)\right|
$$

Thus, $\widetilde{Q}_{S, v}$ for $v \in \mathbb{R}^{d}$ and invertible $S \in \mathbb{R}^{d \times d}$ takes the form

$$
\widetilde{Q}_{S, v}(f)=\frac{1}{|\operatorname{det} S|} \sum_{m \in \mathbb{Z}^{d}} f\left(\Psi\left(S^{-\top}(m+v)\right)\right) \cdot\left|D \Psi\left(S^{-\top}(m+v)\right)\right|
$$

for any function $f:[0,1]^{d} \rightarrow \mathbb{R}$. Notice that $\left|D \Psi\left(S^{-\top}(m+v)\right)\right|$ is zero, if $S^{-\top}(m+v) \notin[0,1]^{d}$.

Algorithm. For a Frolov matrix $B \in \mathbb{R}^{d \times d}$ and any $a>0$ the transformed randomized Frolov quadrature formula $\widetilde{M}_{a, B}$ is the method $\widetilde{Q}_{a \bar{u} B, v}$ with independent $u$ and $v$, uniformly distributed in $\left[1,2^{1 / d}\right]^{d}$ and $[0,1]^{d}$ respectively.

Lemma 6. The method $\widetilde{M}_{a, B}$ is well-defined and unbiased on $L^{1}\left([0,1]^{d}\right)$.

Proof. Let $f \in L^{1}\left([0,1]^{d}\right)$. By the change of variables theorem $f_{0}=f \circ \Psi \cdot|D \Psi|$ 
is also integrable on $[0,1]^{d}$ and satisfies

$$
\widetilde{Q}_{a \bar{u} B, v}(f)=Q_{a \bar{u} B, v}\left(f_{0}\right) \text { and } \quad I_{d}(f)=I_{d}\left(f_{0}\right) .
$$

Thus $\widetilde{M}_{a, B}^{\omega}(f)=M_{a, B}^{\omega}\left(f_{0}\right)$ for any realization $\widetilde{M}_{a, B}^{\omega}$ of $\widetilde{M}_{a, B}$. This yields

$$
\mathbb{E}\left(\widetilde{M}_{a, B}(f)\right)=\mathbb{E}\left(M_{a, B}\left(f_{0}\right)\right)=I_{d}\left(f_{0}\right)=I_{d}(f)
$$

by Lemma 3 ,

The following is our main result. It is important to recall that the number $n$ of function evaluations in $\widetilde{M}_{a, B}$ is of the order $a^{d}$, see Lemma 1 .

Theorem 6. Let $B \in \mathbb{R}^{d \times d}$ be a Frolov matrix and $r, s \in \mathbb{N}$ with $s>d / 2$. Then there is a constant $c>0$ such that for every $a \geq 2^{1 / d}$ and $f \in H^{r, \operatorname{mix}}\left([0,1]^{d}\right)$

$$
\begin{aligned}
& \mathbb{E}\left|\widetilde{M}_{a, B}(f)-I_{d}(f)\right| \leq c a^{-r d-d / 2}(\log a)^{\frac{d-1}{2}}\|f\|_{H^{r, \operatorname{mix}\left([0,1]^{d}\right)}} \quad \text { and } \\
& \sup _{\omega}\left|\widetilde{M}_{a, B}^{\omega}(f)-I_{d}(f)\right| \leq c a^{-r d}(\log a)^{\frac{d-1}{2}}\|f\|_{H^{r, \operatorname{mix}\left([0,1]^{d}\right)}}
\end{aligned}
$$

and for every $a>0$ and $f \in H^{s}\left([0,1]^{d}\right)$

$$
\begin{aligned}
& \mathbb{E}\left|\widetilde{M}_{a, B}(f)-I_{d}(f)\right| \leq c a^{-s-d / 2}\|f\|_{H^{s}\left([0,1]^{d}\right)} \quad \text { and } \\
& \sup _{\omega}\left|\widetilde{M}_{a, B}^{\omega}(f)-I_{d}(f)\right| \leq c a^{-s}\|f\|_{H^{s}\left([0,1]^{d}\right)},
\end{aligned}
$$

where the suprema are taken over all realizations $\widetilde{M}_{a, B}^{\omega}$ of $\widetilde{M}_{a, B}$.

Proof. Remember that $\widetilde{M}_{a, B}^{\omega}(f)=M_{a, B}^{\omega}\left(f_{0}\right)$ for $f \in L^{1}\left(\mathbb{R}^{d}\right), f_{0}=f \circ \Psi \cdot|D \Psi|$ and any realization $\widetilde{M}_{a, B}^{\omega}$ of $\widetilde{M}_{a, B}$. Since $\psi^{\prime}(x)=0$ for $x \notin(0,1)$, we know that $\left.D^{\alpha} f_{0}\right|_{\partial[0,1]^{d}}=0$ for each $\alpha \in\{0, \ldots, r\}^{d}$ and hence $f_{0} \in \stackrel{\circ}{H}^{r, \operatorname{mix}}\left([0,1]^{d}\right) \subseteq \stackrel{\circ}{H}^{r, \text { mix }}\left(\mathbb{R}^{d}\right)$ for each $f \in H^{r, \operatorname{mix}}\left([0,1]^{d}\right)$.

This yields

$\mathbb{E}\left|\widetilde{M}_{a, B}(f)-I_{d}(f)\right|=\mathbb{E}\left|M_{a, B}\left(f_{0}\right)-I_{d}\left(f_{0}\right)\right| \leq c \cdot a^{-r d-d / 2}(\log a)^{\frac{d-1}{2}} \cdot\left\|f_{0}\right\|_{H^{r, \operatorname{mix}}\left(\mathbb{R}^{d}\right)}$

as well as

$$
\sup _{\omega}\left|\widetilde{M}_{a, B}^{\omega}(f)-I_{d}(f)\right|=\sup _{\omega}\left|M_{a, B}^{\omega}\left(f_{0}\right)-I_{d}\left(f_{0}\right)\right| \leq c \cdot a^{-r d}(\log a)^{\frac{d-1}{2}} \cdot\left\|f_{0}\right\|_{H^{r, \operatorname{mix}\left(\mathbb{R}^{d}\right)}},
$$


if $c>0$ is the maximum of the constants of Theorem 2 and Theorem 3 . That proves the first statement, since there is a constant $c_{0}>0$ such that every function $f \in H^{r, \operatorname{mix}}\left([0,1]^{d}\right)$ satisfies $\left\|f_{0}\right\|_{H^{r, \operatorname{mix}\left(\mathbb{R}^{d}\right)}} \leq c_{0}\|f\|_{H^{r, \operatorname{mix}\left([0,1]^{d}\right)}}$.

This can be proven as follows. The partial derivatives of $f_{0}$ take the form

$$
D^{\alpha} f_{0}(x)=\frac{\partial^{\|\alpha\|_{1}}}{\partial x_{1}^{\alpha_{1}} \cdots \partial x_{d}^{\alpha_{d}}} f(\Psi(x)) \cdot \prod_{i=1}^{d} \psi^{\prime}\left(x_{i}\right)=\sum_{\beta_{1}, \ldots, \beta_{d}=0}^{\alpha_{1}, \ldots, \alpha_{d}} D^{\beta} f(\Psi(x)) \cdot S_{\alpha, \beta}(x)
$$

for $\alpha \in\{0,1, \ldots, r\}^{d}$, where $S_{\alpha, \beta}(x)$ is a finite sum of finite products of terms $\psi^{(j)}\left(x_{i}\right)$ with $i \in\{1, \ldots, d\}, j \in\{1, \ldots, r d+1\}$ and does not depend on $f$. It is therefore continuous and bounded by some $c_{\alpha, \beta}>0$. Using the Cauchy inequality

$$
\left|\sum_{i=1}^{\operatorname{dim} v} v_{i}\right|^{2}=\left|\left\langle v,(1, \ldots, 1)^{\top}\right\rangle\right|^{2} \leq\|v\|_{2}^{2} \cdot\left\|(1, \ldots, 1)^{\top}\right\|_{2}^{2}=\operatorname{dim} v \cdot \sum_{i=1}^{\operatorname{dim} v}\left|v_{i}\right|^{2}
$$

for real vectors $v$, we get

$$
\begin{aligned}
& \left\|D^{\alpha} f_{0}\right\|_{L^{2}\left(\mathbb{R}^{d}\right)}^{2} \leq\left(\sum_{\beta_{1}, \ldots, \beta_{d}=0}^{\alpha_{1}, \ldots, \alpha_{d}}\left\|\left(D^{\beta} f \circ \Psi\right) \cdot S_{\alpha, \beta}\right\|_{L^{2}\left([0,1]^{d}\right)}\right)^{2} \\
& \leq\left(\sum_{\beta_{1}, \ldots, \beta_{d}=0}^{\alpha_{1}, \ldots, \alpha_{d}} c_{\alpha, \beta} \cdot\left\|D^{\beta} f \circ \Psi\right\|_{L^{2}\left([0,1]^{d}\right)}\right)^{2} \\
& \left.\stackrel{\text { Cauchy }}{\leq}(r+1)^{d} \sum_{\beta_{1}, \ldots, \beta_{d}=0}^{\alpha_{1}, \ldots, \alpha_{d}} c_{\alpha, \beta}^{2} \cdot\left\|D^{\beta} f \circ \Psi\right\|_{L^{2}([0,1]}^{2}\right) \\
& =(r+1)^{d} \sum_{\beta_{1}, \ldots, \beta_{d}=0}^{\alpha_{1}, \ldots, \alpha_{d}} c_{\alpha, \beta}^{2} \int_{(0,1)^{d}}\left|D^{\beta} f(\Psi(x))\right|^{2} \mathrm{~d} x \\
& =(r+1)^{d} \sum_{\beta_{1}, \ldots, \beta_{d}=0}^{\alpha_{1}, \ldots, \alpha_{d}} c_{\alpha, \beta}^{2} \int_{\Psi\left((0,1)^{d}\right)} \mid D^{\beta} f\left(\left.\Psi\left(\Psi^{-1}(x)\right)\right|^{2} \cdot\left|D \Psi^{-1}(x)\right| \mathrm{d} x\right. \\
& \leq(r+1)^{d} \sup _{x \in(0,1)^{d}}\left|D \Psi^{-1}(x)\right| \sum_{\beta_{1}, \ldots, \beta_{d}=0}^{\alpha_{1}, \ldots, \alpha_{d}} c_{\alpha, \beta}^{2} \cdot\left\|D^{\beta} f\right\|_{L^{2}\left([0,1]^{d}\right)}^{2} \\
& \leq c_{\alpha} \cdot\|f\|_{H^{r, \text { mix }\left([0,1]^{d}\right)}}^{2} \text {, }
\end{aligned}
$$

for some $c_{\alpha}>0$ and

$$
\left\|f_{0}\right\|_{H^{r, \operatorname{mix}}\left(\mathbb{R}^{d}\right)}^{2}=\sum_{\alpha \in\{0,1, \ldots, r\}^{d}}\left\|D^{\alpha} f_{0}\right\|_{L^{2}\left(\mathbb{R}^{d}\right)}^{2} \leq\left(\sum_{\alpha \in\{0,1, \ldots, r\}^{d}} c_{\alpha}\right) \cdot\|f\|_{H^{r, \operatorname{mix}\left([0,1]^{d}\right)}}^{2} .
$$


The second statement is proven in the exact same manner.

A translation of Theorem 6 by means of Lemma 1 shows that the algorithm $\widetilde{M}_{a, B}$ indeed satisfies all the properties (P1) and (P2) and (P3).

Summary. Let $d, r, s \in \mathbb{N}$ with $s>d / 2$ and $B \in \mathbb{R}^{d \times d}$ be a Frolov matrix. Then there is a constant $c>0$ (that may depend on $B$ and $r$ or $s$ ) such that for every $n \in \mathbb{N}$ there is some $a_{n}>0$ so that $\widetilde{M}_{a_{n}, B}$ uses at most $n$ function values of any $f \in L^{1}\left([0,1]^{d}\right)$ and satisfies

$$
\begin{aligned}
& \mathbb{E}\left(\widetilde{M}_{a_{n}, B}(f)\right) \quad=I_{d}(f), \\
& \mathbb{E}\left|\widetilde{M}_{a_{n}, B}(f)-I_{d}(f)\right| \leq c n^{-r-1 / 2}(\log n)^{\frac{d-1}{2}}\|f\|_{H^{r, \operatorname{mix}([0,1] d)}}, \quad \text { if } n>1, \\
& \sup _{\omega}\left|\widetilde{M}_{a_{n}, B}^{\omega}(f)-I_{d}(f)\right| \leq c n^{-r}(\log n)^{\frac{d-1}{2}}\|f\|_{H^{r, \text { mix }\left([0,1]^{d}\right)}}, \quad \text { if } n>1 \text {, } \\
& \mathbb{E}\left|\widetilde{M}_{a_{n}, B}(f)-I_{d}(f)\right| \leq c n^{-s / d-1 / 2}\|f\|_{H^{s}\left([0,1]^{d}\right)}, \quad \text { and } \\
& \sup _{\omega}\left|\widetilde{M}_{a_{n}, B}^{\omega}(f)-I_{d}(f)\right| \leq c n^{-s / d}\|f\|_{H^{s}\left([0,1]^{d}\right)} \text {. }
\end{aligned}
$$

Proof. Let $c_{1}=\left(\|B\|_{1}+1\right)^{d} \geq 1$ be the constant of Lemma 1 and $c_{2}>0$ be the constant of Theorem 6 . For $n \geq 4 c_{1}$, we set $a_{n}=\left(n /\left(2 c_{1}\right)\right)^{1 / d} \geq 2^{1 / d}$. By Lemma 1, the Monte Carlo method $\widetilde{M}_{a_{n}, B}$ uses no more than $2 c_{1} \cdot a_{n}^{d}=n$ function values of $f$. For $n<4 c_{1}$ we choose $a_{n}>0$ small enough such that the only node of $\widetilde{M}_{a_{n}, B}$ is zero. The method $\widetilde{M}_{a_{n}, B}$ is thus unbiased for all $n \in \mathbb{N}$ and uses at most $n$ function values.

Theorem 6 yields for $n \geq 4 c_{1}$ and thus $a_{n} \geq 2^{1 / d}$ that

$$
\begin{aligned}
& \mathbb{E}\left|\widetilde{M}_{a_{n}, B}(f)-I_{d}(f)\right| \leq c_{2}\left(\left(n / 2 c_{1}\right)^{1 / d}\right)^{-r d-d / 2}\left(\log \left(n / 2 c_{1}\right)^{1 / d}\right)^{\frac{d-1}{2}}\|f\|_{H^{r, \operatorname{mix}\left([0,1]^{d}\right)}} \\
& \stackrel{c_{1} \geq 1}{\leq} c_{2} d^{-\frac{d-1}{2}}\left(2 c_{1}\right)^{r+1 / 2} n^{-r-1 / 2}(\log n)^{\frac{d-1}{2}}\|f\|_{H^{r, \operatorname{mix}\left([0,1]^{d}\right)}}, \\
& \mathbb{E}\left|\widetilde{M}_{a_{n}, B}(f)-I_{d}(f)\right| \leq c_{2}\left(\left(n / 2 c_{1}\right)^{1 / d}\right)^{-s-d / 2}\|f\|_{H^{s}\left([0,1]^{d}\right)} \\
& =c_{2}\left(2 c_{1}\right)^{s / d+1 / 2} n^{-s / d-1 / 2}\|f\|_{H^{s}\left([0,1]^{d}\right)}, \\
& \left.\sup _{\omega}\left|\widetilde{M}_{a_{n}, B}^{\omega}(f)-I_{d}(f)\right| \leq c_{2}\left(\left(n / 2 c_{1}\right)^{1 / d}\right)^{-r d}\left(\log \left(n / 2 c_{1}\right)\right)^{1 / d}\right)^{\frac{d-1}{2}}\|f\|_{\left.\left.H^{r, \operatorname{mix}([0,1]}\right]^{d}\right)} \\
& \stackrel{c_{1} \geq 1}{\leq} c_{2} d^{-\frac{d-1}{2}}\left(2 c_{1}\right)^{r} n^{-r}(\log n)^{\frac{d-1}{2}}\|f\|_{H^{r, \operatorname{mix}\left([0,1]^{d}\right)}}, \\
& \sup _{\omega}\left|\widetilde{M}_{a_{n}, B}^{\omega}(f)-I_{d}(f)\right| \leq c_{2}\left(\left(n / 2 c_{1}\right)^{1 / d}\right)^{-s}\|f\|_{H^{s}\left([0,1]^{d}\right)} \\
& =c_{2}\left(2 c_{1}\right)^{s / d} n^{-s / d}\|f\|_{H^{s}\left([0,1]^{d}\right)} \text {. }
\end{aligned}
$$


This shows that the stated bounds hold for the maximum $c$ of the constants $c_{2} d^{-\frac{d-1}{2}}\left(2 c_{1}\right)^{r+1 / 2}, c_{2}\left(2 c_{1}\right)^{s / d+1 / 2}$ and possibly larger constants that result from the cases $n=1, \ldots,\left\lfloor 4 c_{1}\right\rfloor$.

\section{References}

[1] K. K. Frolov: Upper Error Bounds for Quadrature Formulas on Function Classes. Soviet Mathematics Doklady 17/6, pp. 1665-1669, 1976.

[2] F. J. Hickernell: My dream quadrature rule. J. Complexity 19, pp. 420-427, 2003.

[3] H. Koch: Number Theory: Algebraic Numbers and Functions. Graduate Studies in Mathematics. American Mathematical Society, Providence, 2000.

[4] E. Novak: Deterministic and Stochastic Error Bounds in Numerical Analysis. Lecture Notes in Mathematics 1349, Springer, 1988.

[5] E. Novak and H. Woźniakowski: Tractability of Multivariate Problems II: Standard Information for Functionals. European Mathematical Society, 2010 .

[6] M. M. Skriganov: Constructions of uniform distributions in terms of geometry of numbers. Algebra i Analiz 6, 200-230, 1994.

[7] V.N.Temlyakov: Approximation of Periodic Functions. Computational Mathematics and Analysis Series. Nova Science Publishers, New York, 1993.

[8] V. N. Temlyakov: Cubature formulas, discrepancy, and nonlinear approximation. Journal of Complexity 19, pp. 352-391, 2003.

[9] M. Ullrich: On "Upper error bounds for quadrature formulas on function classes" by K. K. Frolov. Manuscript, http://arxiv.org/abs/1404.5457, 2014.

[10] M. Ullrich and T. Ullrich: The role of Frolov's cubature formula for functions with bounded mixed derivative. Manuscript, http://arxiv.org/abs/1503.08846, 2015. 